\title{
Epidemiology of Raoultella species in the context of human infections: A 10-year retrospective study in a tertiary-care hospital in Hungary
}

\author{
Márió Gajdács* \\ Department of Pharmacodynamics and Biopharmacy, Faculty of Pharmacy, University of Szeged, 6720 Szeged, Dóm tér 10., Hungary
}

\begin{abstract}
Introduction: Raoultella species are Gram-negative non-motile, aerobic, oxidase-negative and catalase-positive rods that are members of the Enterobacterales order that are infrequently described as significant pathogens in human disease.

Objectives of the study: To report the incidence and the susceptibility levels of Raoultella species from clinical samples at a tertiary-care center in Hungary, during a 10-year study period (2008-2017).

Method: Data collection was performed electronically by searching the records of the laboratory information system (LIS), regarding the samples with significant colony counts for Raoultella spp.

Results: N=40 individual Raoultella isolates were identified (4.0 $\pm 2.8 /$ year, range: 0-9 isolates; highest in 2015, lowest in 2010 and 2011). Raoultella species were isolated from the following sample types: urine (catheterized and midstream): $\mathrm{n}=18$, blood cultures: $\mathrm{n}=12$, stool: $\mathrm{n}=6$ and abscesses: $\mathrm{n}=4.36$ out of 40 isolates were $R$. ornitbinolytica, while the remaining $\mathrm{n}=4$ were $R$. planticola. All of the tested strains were susceptible to the group of $\beta$-lactam antibiotics.

Conclusion: Raoultella species should be considered as emerging pathogens in the context of human infections, especially in older individuals in people with severe immunosuppression. The results suggest that in our local settings, there was-on average-one isolation of Raoultella every three months from various clinical samples and $\beta$-lactams may be considered safe choices for empiric therapy.
\end{abstract}

\section{Introduction}

Raoultella species are Gram-negative non-motile, aerobic, oxidase-negative and catalase-positive rods that are members of the Enterobacterales order (gut bacteria) [1,2]. The genus Raoultella was previously designated to the genus Klebsiella (as cluster II; e.g., $K$. ornithinolytica), but after extensive advancement in phylogenetic methods (16S rRNA and rDNA sequencing, bacterial RNA polymerase $\beta$ subunit [rpoB] sequence-analysis), the genus Klebsiella was revised and the independent genus Raoultella was created in 2001 (named after Didier Raoult, a French bacteriologist) [3,4]. R. ornithinolytica is the most abundantly found both in clinical samples and in nature (in aquatic environments, soil and in different animals, such as fish, insects and ticks); however $R$. terrigena, $R$. planticola and $R$. trevisanii are also important members of the genus [2-4].

The existing literature on Raoultella infections in the context of human infections is scarce, as the incidence of these pathogens in clinically-relevant syndromes in low [5]. Most existing reports on these bacteria are associated with infections of the gastro-intestinal, hepatobiliary, urological and respiratory systems, while bloodstreaminfections, osteo-articular, soft tissue and ear-nose-throat (ENT) infections have also been published [6-11]. It should be noted, that the difficulty in the adequate identification (especially in low-resource settings) may be partly blamed for the infrequent characterization of these bacteria as significant pathogens [12]. Some reports even suggest that around $20 \%$ of Raoultella species are misidentified as Klebsiella (predominantly K. oxytoca) [13]. Nevertheless, with the advent of novel diagnostic modalities in routine use (such as whole-genome sequencing [WGS] and matrix-assisted laser desorption/ionization time-of-flight mass spectrometry [MALDI-TOF MS]) and reports on the emergence of multidrug-resistant strains of Raoultella, the clinical role of this genus should not be underestimated [14]. The aim of this study was to report the incidence and the susceptibility levels of Raoultella species from clinical samples at a tertiary-care center in Hungary, during a 10year study period (2008-2017).

\section{Materials and methods}

\section{Study design and data collection}

The present retrospective study was carried out using microbiological data collected corresponding to the time period between 2008.01.01.2017.12.31., at the Institute of Clinical Microbiology, University of Szeged. The Institute serves as the dedicated diagnostic institute of the Albert Szent-Györgyi Clinical Center, which is a 1,820-bed

*Correspondence to: Márió Gajdács, Department of Pharmacodynamics and Biopharmacy, Faculty of Pharmacy, University of Szeged, 6720 Szeged, Dóm tér 10., Hungary, E-mail: gajdacs.mario@pharm.u-szeged.hu

Key words: Raoultella, Enterobacterales, human infections, retrospective, epidemiology, clinical, microbiology

Received: November 30, 2019; Accepted: December 11, 2019; Published: December 16, 2019 
primary- and tertiary-care teaching hospital in the Southern Great Plain of Hungary (with an estimated population of over 400,000 people, based on data from 2017) [15]. Data collection was performed electronically by searching the records of the laboratory information system (LIS), regarding the samples with significant colony counts for Raoultella spp. ( $>10^{5} \mathrm{CFU} / \mathrm{mL}$ for urine samples, while $>10^{3}$ in case of other types of clinically-relevant samples; however, this was subject to interpretation by the senior clinical microbiologists, based on the information provided on the clinical request forms for the microbiological analysis and international guidelines) [16]. Only the first isolate per patient was included in the study; however, isolates with different antibiotic-susceptibility patterns from the same patient were considered as different individual isolates. To evaluate the demographic characteristics of these infections, patient data was also collected, which was limited to sex, age at sample submission, and inpatient/outpatient status [16]. The study was deemed exempt from ethics review by the Institutional Review Board and informed consent was not required as data anonymity was maintained.

\section{Sample processing and identification}

The processing of relevant samples arriving to the Institute of Clinical Microbiology was carried out according to guidelines in routine clinical bacteriology. Between 2008-2012, the BD Bactec (Beckton Dickinson, Franklin Lakes, NJ, USA) detection system was employed for the incubation of blood culture bottles, whilst from 2013 onwards, the BacT/ALERT 3D (bioMérieux, Marcy-l'Étoile, France) detection system was used. Blood culture bottles were incubated for 5 days ( 21 days, if endocarditis was suspected) in the abovementioned detection systems [17]. The processing of urine samples was as follows: $10 \mu \mathrm{L}$ of each un-centrifuged urine sample was cultured on UriSelect chromogenic agar plates (Bio-Rad, Berkeley, CA, USA) with a calibrated loop, according to the manufacturer's instructions and incubated at $37^{\circ} \mathrm{C}$ for $24-48 \mathrm{~h}$, aerobically [15]. The workup of faecal samples was performed on the appropriate non-selective and selective media. If the relevant pathogens presented in significant colony count, the plates were passed on for further processing.

Between 2008-2012, presumptive phenotypic (biochemical reaction-based) methods and VITEK 2 ID (bioMérieux, Marcy-l'Étoile, France) were used for bacterial identification, while after 2013, this was complemented by matrix-assisted laser desorption/ionization time-of-flight mass spectrometry (MALDI-TOF MS; Bruker Daltonik Gmbh. Gr., Bremen, Germany). Bacterial cells were transferred to a stainless-steel target. An on-target extraction was performed by adding $1 \mathrm{ml}$ of $70 \%$ formic acid prior to the matrix. After drying at ambient temperature, the cells were covered with $1 \mathrm{ml}$ matrix ( $\alpha$-cyano-4hydroxy cinnamic acid in 50\% acetonitrile/2.5\% trifluoro-acetic acid). Mass spectrometry was performed by the Microflex MALDI Biotyper (Bruker Daltonics, Bremen, Germany) in positive linear mode across the $\mathrm{m} / \mathrm{z}$ range of 2 to $20 \mathrm{kDa}$; for each spectrum, 240 laser shots at $60 \mathrm{~Hz}$ in groups of 40 shots per sampling area were collected [18]. The MALDI Biotyper RTC 3.1 software (Bruker Daltonics) and the MALDI Biotyper Library 3.1 were used for spectrum analysis.

\section{Antimicrobial susceptibility testing (AST)}

Antimicrobial susceptibility testing (AST) was performed using the Kirby-Bauer disk diffusion method and when appropriate, E-test (Liofilchem, Abruzzo, Italy) on Mueller-Hinton agar (MHA) plates. The interpretation of the results was based on EUCAST breakpoints for Enterobacteriaceae (Enterobacterales). The following antibiotics were tested: amoxicillin/clavulanic acid, piperacillin/tazobactam, ceftazidime, ceftriaxone, cefepime, imipenem, meropenem, gentamicin, tobramycin, amikacin, norfloxacin, ciprofloxacin, levofloxacin and sulfamethoxazole/trimethoprim. During data analysis, intermediatelysusceptible results were grouped with and reported as resistant. If extended-spectrum beta-lactamase (ESBL)-production was suspected, detection was carried out based on EUCAST recommendations, using AmpC-ESBL Detection Set (MAST Diagnostica $\mathrm{GmbH}$, Reinfeld, Germany), according to the manufacturer's instructions. Staphylococcus aureus ATCC 29213, Enterococcus faecalis ATCC 29212, Proteus mirabilis ATCC 35659, Escherichia coli ATCC 25922, Klebsiella pneumoniae ATCC 700603 and Pseudomonas aeruginosa ATCC 27853 were used as quality control strains [16].

\section{Statistical analysis}

Descriptive statistical analysis (including means or medians with ranges and percentages to characterize data) was performed using Microsoft Excel 2013 (Redmond, WA, Microsoft Corp.).

\section{Results}

During the 10-year study period, a total of 40 individual Raoultella isolates were identified (4.0 22.8/year, range: 0-9 isolates; highest in 2015, lowest in 2010 and 2011). The number of isolates in the first half of the surveillance period (2008-2012) was $n=12$, while for 2013-2017, this number was $n=28.32$ out of 40 isolates were from samples originating from inpatient departments. Raoultella species were isolated from the following sample types: urine (catheterized and midstream): $n=18$, blood cultures: $n=12$, stool: $n=6$ and abscesses: $n=4$. 36 out of 40 isolates were $R$. ornithinolytica, while the remaining $n=4$ were $R$. planticola. The affected patients presented with a pronounced male dominance ( 29 out of 40 ); the age distribution of patients was the following: $0-5$ years: $n=8,6-35$ years: $n=2 \quad 36-59$ years: $n=12$ and 60 years of age or older: $n=18$. The indications specified on the sample submission forms were the following: diseases of the urinary system (infection, anatomical abnormality): $n=18$, cardiovascular illness: $n=5$, abdominal pain: $n=5$, infection (not specified): $n=3$, diseases of the central nervous system (CNS): $n=2$, sepsis: $n=2$, pneumonia: $n=2$ and other: $\mathrm{n}=3$.

All of the tested strains were susceptible to the group of $\beta$-lactam antibiotics (40 out of 40; from ampicillin to meropenem, respectively), while $8 / 40$ of isolates were resistant to norfloxacin, $6 / 40$ to ciprofloxacin and levofloxacin, $5 / 40$ to sulfamethoxazole/trimethoprim and 2/60 to the tested aminoglycosides, respectively. No ESBL- or AmpC-producing strain was detected during the study period.

\section{Discussion}

The present study reports on the epidemiological features of Raoultella infections at a tertiary-care hospital in Hungary over a period of a decade (2008-2017). Due to the developments in diagnostic technologies in routine clinical microbiology (e.g., MALDI-TOF MS), the prevalence of bacterial species that were previously considered as rare will most probably increase, which is reflected in the increase in the interest towards these bacteria in the literature [1-11,19]. The clinical role and prognosis of Raoultella infections highly depend on the health status of the patient: the risk of sequelae is higher in older, co-morbid patient with invasive infections, therefore the awareness of the clinicians regarding the epidemiology and susceptibility-levels of these bacteria is of utmost importance [20-22]. In case of those patients, the initiation of prompt antibiotic therapy is crucial for recovery, while in immunocompetent patients, symptoms may resolve with supportive 
therapy as well [1-8]. The virulence factors of Raoultella are thought to be similar to the ones of Klebsiella species due to their phylogenic similarities, however, no such correlation was found in vivo [12,23].

Based on the findings in the literature, Raoultella species are usually broadly sensitive to most antibiotics against Gram-negative bacteria: this was demonstrated in our study as well, where all $\beta$-lactams may be considered as safe choices for empiric therapy [1-11]. Other reports in the literature have also highlighted $\beta$-lactams as safe alternatives for therapy, due to very low resistance levels. However, it should be remembered that these bacteria (similarly to Klebsiella spp.) are intrinsically resistant to ampicillin, due to a chromosomally-encoded $\beta$-lactamase enzyme [12,23]. Nonetheless, MDR strains of Raoultella have been described, producing a variety of $\beta$-lactamases (Ambler class A, B and D), including SHV-, TEM- and CTX-M-type extendedspectrum $\beta$-lactamases (ESBLs) and these bacteria have been reported as potential reservoirs for carbapenemase and colistin-resistance genes [24-28].

The limitations of this study should be reported: the study was retrospective, thus the symptoms of individual patients affected by these infections could not be recorded in real-time. Additionally, the molecular characterization of resistance determinants in the mentioned isolates was not performed; therefore non-susceptibility was characterized by phenotypic methods only [15].

\section{Conclusions}

Although infrequently isolated (or identified), Raoultella species should be considered as emerging pathogens in the context of human infections, especially in older individuals in people with severe immunosuppression. The results suggest that in our local settings, there was-on average-one isolation of Raoultella every three months from various clinical samples and $\beta$-lactams may be considered safe choices for empiric therapy of these infections, taking into account that the emergence of resistant mutants is possible. The surveillance of rarely isolated species over long periods of time may help ascertain their true clinical role in human infections.

\section{Author contributions}

M.G. conceived and designed the study, performed data collection and analysis, wrote and revised the full paper.

\section{Funding}

This research received no external funding.

\section{Acknowledgments}

M.G. was supported by the National Youth Excellence Scholarship [Grant Number NTP-NTFÖ-18-C-0225] and the ESCMID Mentorship and Observership Programme.

\section{Conflicts of interest}

The author declares no conflicts of interest, monetary or otherwise.

\section{References}

1. Adeolu M, Alnajar S, Naushad SS, Gupta R (2016) Genome-based phylogeny and taxonomy of the "Enterobacteriales": proposal for Enterobacterales ord. nov. divided into the families Enterobacteriaceae, Erwiniaceae fam. nov., Pectobacteriaceae fam. nov., Yersiniaceae fam. nov., Hafniaceae fam. nov., Morganellaceae fam. nov., and Budviciaceae fam. nov. Int J Syst Evol Microbiol 66: 5575-5599.

2. Chun S, Yun JW, Huh HJ, Lee NY (2015) Clinical characteristics of Raoultella ornithinolytica bacteremia. Infection 43: 59-64
3. Drancourt M, Bollet C, Carta A, Rousselier P (2001) Phylogenetic analyses of Klebsiella species delineate Klebsiella and Raoultella gen. nov., with description of Raoultella ornithinolytica comb. nov., Raoultella terrigena comb. nov. and Raoultella planticola comb. nov. Int J Syst Evol Microbiol 51: 925-932.

4. Kanki M, Yoda T, Tsukamoto T, Shibata T (2002) Klebsiella pneumoniae produces no histamine: Raoultella planticola and Raoultella ornithinolytica strains are histamine producers. Appl Environ Microbiol 68: 3462-3466.

5. Seng P, Boushab BM, Romain F, Gouriet F, Bruder N, et al. (2016) Emerging role of Raoultella ornithinolytica in human infections: a series of cases and review of the literature. Int J Infect Dis 45: 65-71. [Crossref]

6. Sekowska A (2017) Raoultella spp.-clinical significance, infections and susceptibility to antibiotics. Folia Microbiol (Praha) 62: 221-227.

7. Buyukcam A, Liste U, Bicakcigil A, Kara A, Sancak B (2019) A case of Raoultella ornithinolytica urinary tract infection in a pediatric patient. $J$ Infect Chemother 25 467-469.

8. Sener D, Cokhras H, Camcioglu Y, Akcakaya N, Cakir E (2011) Raoultella infection causing fever of unknown origin. Ped Infect Dis J 30: 1122-1123.

9. Haruki Y, Hagiya H, Sakuma A, Murase T, Sugiyama T, et al. (2014) Clinical characteristics of Raoultella ornithinolytica bacteremia: a case series and literature review. J Infect Chemother 20: 589-591. [Crossref]

10. Van Cleve JR, Boucher BA, Smith DV, Croce MA (2018) Ventilator associated pneumonia caused by Raoultella ornithinolytica in two immunocompetent trauma patients. Respir Med Case Rep 24: 135-137.

11. Podschun R, Acktun H, Okpara J, Linderkamp O, Ullmann U, et al. (1998) Isolation of Klebsiella planticola from newborns in a neonatal ward. J Clin Microbiol 36: 23312332. [Crossref]

12. Park JS, Hong KH, Lee HJ, Choi SH, Song SH, et al. (2011) Evaluation of three phenotypic identification systems for clinical isolates of Raoultella ornithinolytica. $J$ Med Microbiol 60: 492-499.

13. Mori M, Ohta M, Agata N, Kido N, Arakawa Y, et al. (1989) Identification of species and capsular types of Klebsiella clinical isolates, with special reference to Klebsiella planticola. Microbiol Immunol 33: 887-895.

14. Gajdács M, Urbán E (2019) Resistance trends and epidemiology of Citrobacterenterobacter-serratia in urinary tract infections of inpatients and outpatients (RECESUTI): A 10-Year Survey. Medicina 55: 285.

15. Gajdács M, Burián K, Terhes G (2019) Resistance levels and epidemiology of nonfermenting gram-negative bacteria in urinary tract infections of inpatients and outpatients (RENFUTI): A 10-year epidemiological snapshot. Antibiotics 8: 143.

16. Gajdács M, Urbán E (2019) The relevance of anaerobic bacteria in brain abscesses: A ten-year retrospective analysis (2008-2017). Infect Dis 51: 779-781.

17. Schubert S, Kostrzewa M (2017) MALDI-TOF MS in the microbiology laboratory: Current trends. Curr Issues Mol Biol 23: 17-20.

18. de Jong E, de Jong AS, Smidts-van den Berg N, Rentenaar RJ (2013) Differentiation of Raoultella ornithinolytica/planticola and Klebsiella oxytoca clinical isolates by matrixassisted laser desorption/ionization-time of flight mass spectrometry. Diagn Microbiol Infect Dis 75: 431-433.

19. Lam PW, Tadros M, Fong IW (2018) Mandibular osteomyelitis due to Raoultella species. JMM Case Rep 5: e005140.

20. Gajdács M, Szabó A (2019) Physicians' opinions towards antibiotic use and resistance in the southeastern region of Hungary.

21. Gajdács M (2019) Intravenous or oral antibiotic therapy: Sophie’s choice? Gen Int Med Clin Innov 4: 1-2.

22. Tominaga T (2018) Rapid detection of Klebsiella pneumoniae, Klebsiella oxytoca, Raoultella ornithinolytica and other related bacteria in food by lateral-flow test strip immunoassays. J Microbiol Methods 147: 43-49.

23. Demiray T, Koroglu M, Ozbek A, Altindis M (2016) A rare cause of infection, Raoultella planticola: emerging threat and new reservoir for carbapenem resistance. Infection 44: 713-7.

24. Wang X, Wang Y, Zhou Y, Wang Z, Wang Y, et al. (2019) Emergence of colistin resistance gene mcr-8 and its variant in Raoultella ornithinolytica. Front Microbiol 10: 228.

25. Zurfluh K, Hachler H, Nuesch-Inderbinen M, Stephan R (2013) Characteristics of extended-spectrum beta-lactamase- and carbapenemase-producing Enterobacteriaceae Isolates from rivers and lakes in Switzerland. Appl Environ Microbiol 79: 3021-3026. 
Gajdács M (2019) Epidemiology of Raoultella species in the context of human infections: A 10-year retrospective study in a tertiary-care hospital in Hungary

26. Abid IN (2016) Emergence of Raoultella ornithinolytica producing AmpC-betalactamases in the different clinical specimens. J Nat Res Sci 6: 124-129.
27. Castanheira M, Deshpande LM, DiPersio JR, Kang J, Weinstein MP, et al. (2009) First descriptions of blaKPC in Raoultella spp. (R. planticola and R. ornithinolytica): Report from the SENTRY antimicrobial surveillance program. J Clin Microbiol 47: 4129-4130.

Copyright: $@ 2019$ Gajdács M. This is an open-access article distributed under the terms of the Creative Commons Attribution License, which permits unrestricted use, distribution, and reproduction in any medium, provided the original author and source are credited. 